\title{
Victor Klemperer: il coraggio del diario come forma di resistenza
}

\author{
di Anna Ruchat and Maurizio Guerri
}

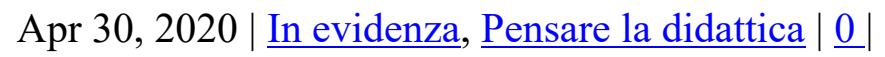

Dresda bombardata durante la Seconda guerra mondiale - Foto conservata nei Bundesarchiv, Bild 183-Z0309-310 / G. Beyer / CC-BY-SA 3.0, CC BY-SA 3.0 de, Link

\begin{abstract}
Victor Klemperer è uno dei grandi testimoni della tragedia tedesca raccontata dall'interno e i suoi diari dal 1933 al 1945 sono un testo imprescindibile per chi voglia capire il Novecento. L'antologia dai Diari di Klemperer qui presentata offre un materiale utilissimo per i docenti di storia, filosofia, di letteratura che intendano costruire dei percorsi didattici a partire da testi diaristici o comunque da materiali coevi alla storia della Germania nazista.

La nota bibliografica in conclusione permette la costruzione di percorsi di approfondimento e di comparazione, come nel caso dell'opera di Primo Levi.
\end{abstract}

«Le parole possono essere piccole dosi di arsenico, le inghiotti senza rendertene conto, sembrano non avere alcun effetto, e dopo qualche tempo l'avvelenamento è avvenuto.»

Victor Klemperer

Victor Klemperer, infaticabile scrittore di diari - la sua opera diaristica inizia nel 1897, quando l'autore ha 16 anni, termina nel 1959, qualche mese prima della sua morte e ammonta a molte migliaia di pagine - è il grande testimone della tragedia tedesca raccontata dall'interno, dal punto di vista di un ebreo privilegiato, costantemente minacciato, che scruta il presente con occhio vigile, impietoso verso se stesso prima ancora che verso gli altri, sempre animato dalla ferma volontà di capire e di lasciare a chi verrà dopo un documento capace di colmare nel dettaglio le inevitabili lacune delle sintesi storiche. Per questo i suoi diari dal 1933 al 1945, (pubblicati in Italia nel 2000 da Mondadori) continuano a essere un testo imprescindibile per chi voglia capire il Novecento tedesco.

Nel febbraio del 1933, quando i nazionalsocialisti vanno al potere, Victor Klemperer si trova a Dresda, dove rimarrà - con la breve interruzione della discesa in Baviera dopo il bombardamento di Dresda - per tutta la guerra e anche dopo e dove è sepolto. Klemperer è un professore tedesco di cultura europea e di origini ebraiche; storico della letteratura francese presso la Technische Hochschule di Dresda, è allievo di Voßler, collega di Auerbach e di Curtius, ma non sono gli studi a costituire il filo rosso della sua esistenza, bensì una ferrea volontà di integrazione culturale e sociale. Ultimo dei sette figli del rabbino capo della Comunità riformata berlinese, Klemperer aveva 
sposato una donna ariana, si era convertito, laico, alla fede protestante, inoltre aveva partecipato alla Prima guerra mondiale, compiendo così, come e più dei fratelli, tutti i passi richiesti a un ebreo che volesse vedersi riconosciuta l'appartenenza al popolo tedesco. L'investimento dedicato negli anni all'assimilazione e la fiducia nella natura e nella cultura tedesca impediscono a Klemperer di andarsene dalla Germania e legano il suo destino a quello della città di Dresda, ma non lo accecano: fin dal febbraio 1933 egli è in grado di capire che l'ascesa al potere di Hitler significa innanzitutto una sconfitta irreparabile per la cultura tedesca ed europea, e più ancora per quell'ideale di umanesimo laico sul quale aveva costruito la propria identità intellettuale.

I diari 1933-45 (usciti in italiano nel 2000 con il titolo Testimoniare fino all'ultimo) sono la cronaca di questa sconfitta, la testimonianza sismografica e sempre più consapevole a mano a mano che il tempo passa, delle mutazioni che avvengono nel dettaglio all'interno della società tedesca e di cui, come lui stesso sottolinea più volte, non ci sarà traccia nelle grandi sintesi storiche. Più recalcitrante invece, incredula talvolta, costernata, ancorché lucidissima è la costatazione dei termini reali in cui avvengono l'isolamento, la selezione e la deportazione degli ebrei.

«Si sa molto dell'orrore dei lager, che Klemperer non attraversa di persona per una serie di circostanze», scrive Antonio Moresco nell'introduzione al diario del 1945 (pubblicato a cura di chi scrive da Scheiwiller nel 2009), poco o niente si sa invece di quello che è stato nel dettaglio, nel quotidiano, l'avvento del regime nazista. Klemperer «registra» scrive ancora Moresco, «nel suo farsi e fin nei minimi particolari quotidiani la vita dell'enorme e impazzito organismo ferito a morte, dove ogni rotellina cerca di funzionare fino all'ultimo all'interno della catastrofe, ci mostra ciò che è successo a un intero paese sprofondato nel delirio di superiorità e di potenza e nell'abiezione. Vengono registrati, fin nelle loro più intime nervature, gli scricchiolii interni e gli spasmi dell'enorme, spaventoso edificio che sta per crollare».[1]

Anno della svolta radicale, il 1945, ha finestre spalancate sul "prima" e lascia intravedere il "dopo" ma contiene anche una riflessione a caldo sull'opportunità o meno di pubblicare i diari 1933-45, sulla necessità di scorporarne le riflessioni sulla lingua del Terzo Reich, annotate via via fin dal 1933 - e raccolte effettivamente poco dopo (1947) nel fondamentale volume LTI, La lingua del Terzo Reich (Giuntina 1998) -, sul valore stesso dei diari come strumento di conoscenza, che rimane unica in tutto il corpus klempereriano. Dalle due pubblicazioni dei diari in italiano, estrapoliamo dei frammenti che spaziano dal 1933 con l'affacciarsi delle persecuzioni antiebraiche a quando, a liberazione avvenuta, Klemperer si reinstalla con la moglie Eva nella casa di Dresda. 


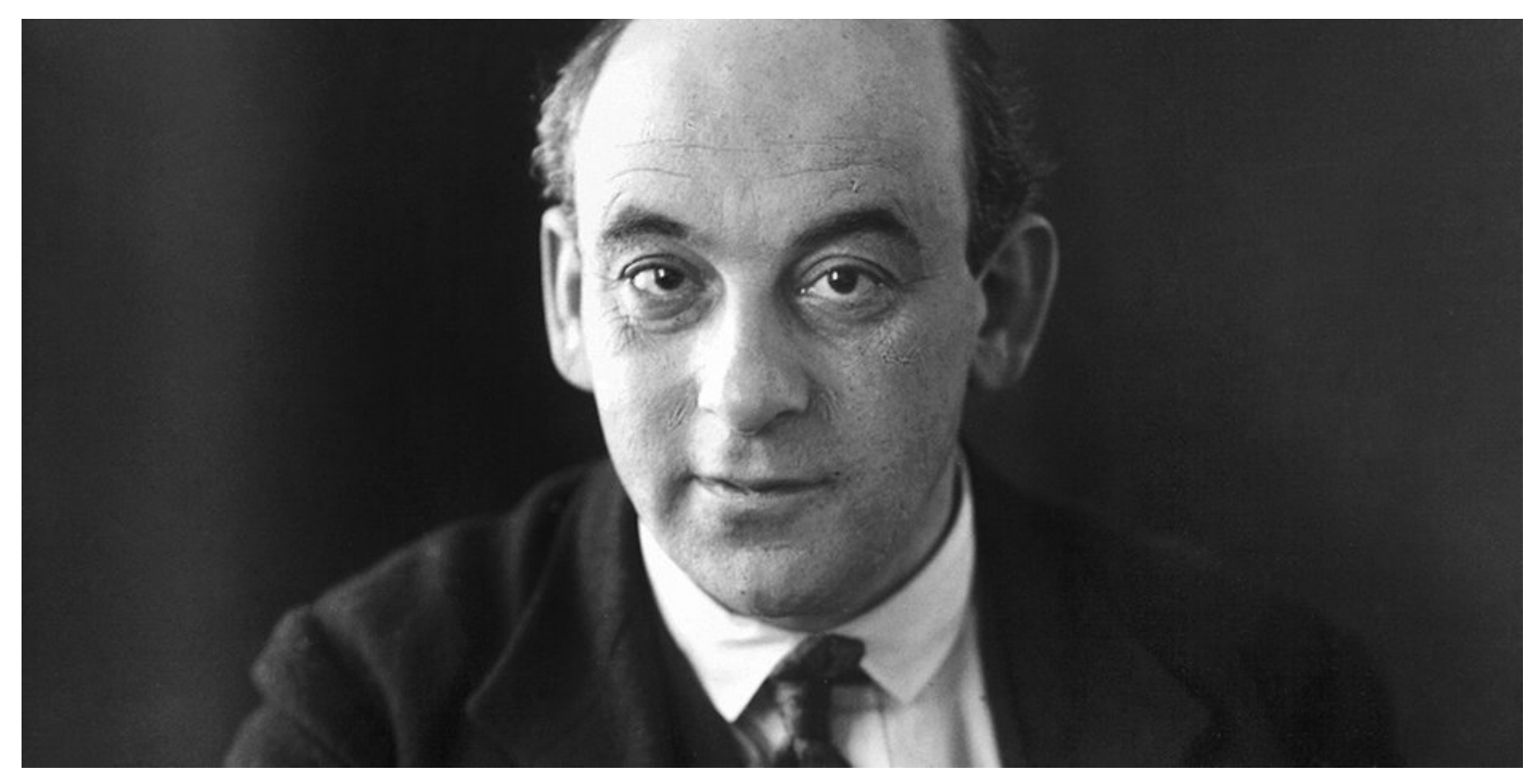

Victor Klemperer intorno al 1930. Foto di Ursula Richter (1886-1946) - BZ vom 13.6.2017 (ursprünglich: Sächsische Staats- und Universitätsbibliothek (SLUB)/Deutsche Fotothek), Gemeinfrei, https://commons.wikimedia.org/w/index.php?curid=69960800

L'antologia dai Diari di Klemperer qui presentati offre un materiale fondamentale - e ancora troppo poco conosciuto - per i docenti di storia, filosofia, di letteratura che intendano costruire dei percorsi didattici a partire da testi diaristici o comunque da materiali coevi alla storia della Germania nazista. Come sottolineato in precedenza, un aspetto centrale dei diari di Klemperer è rappresentato dalla prospettiva "privilegiata" di osservatore: cittadino tedesco (ripudiato dalla Germania) e docente (altrettanto rifiutato) è riuscito ad attraversare tutto il periodo del regime nazista con una crescente consapevolezza del ruolo di testimone che stava svolgendo. Nei Diari di Klemperer, la prospettiva storica è rovesciata rispetto a una concezione evenemenziale della storia: i nomi celebri e $\mathrm{i}$ grandi eventi nelle pagine dei suoi diari rimangono sullo sfondo, oppure quando affiorano sono sospinti da una moltitudine di aspetti della vita quotidiana che d'altro canto in precedenza ne hanno prefigurato l'avvento. I Diari di Klemperer, quindi, consentono di entrare con uno sguardo micrologico nella quotidianità della società tedesca sotto il nazionalsocialismo, vista da un intellettuale che si considerava tedesco, ma che dalla comunità etnica e intellettuale dei tedeschi era stato a forza escluso e solo grazie all'amore della moglie Eva e alla buona sorte si è potuto salvare: "Jaweh!" esclama e poi scrive l'ateo Victor Klemperer quando, subito dopo i bombardamenti su Dresda, scopre che lui, la moglie, e la valigia che contiene i manoscritti dei diari sono rimasti illesi. 

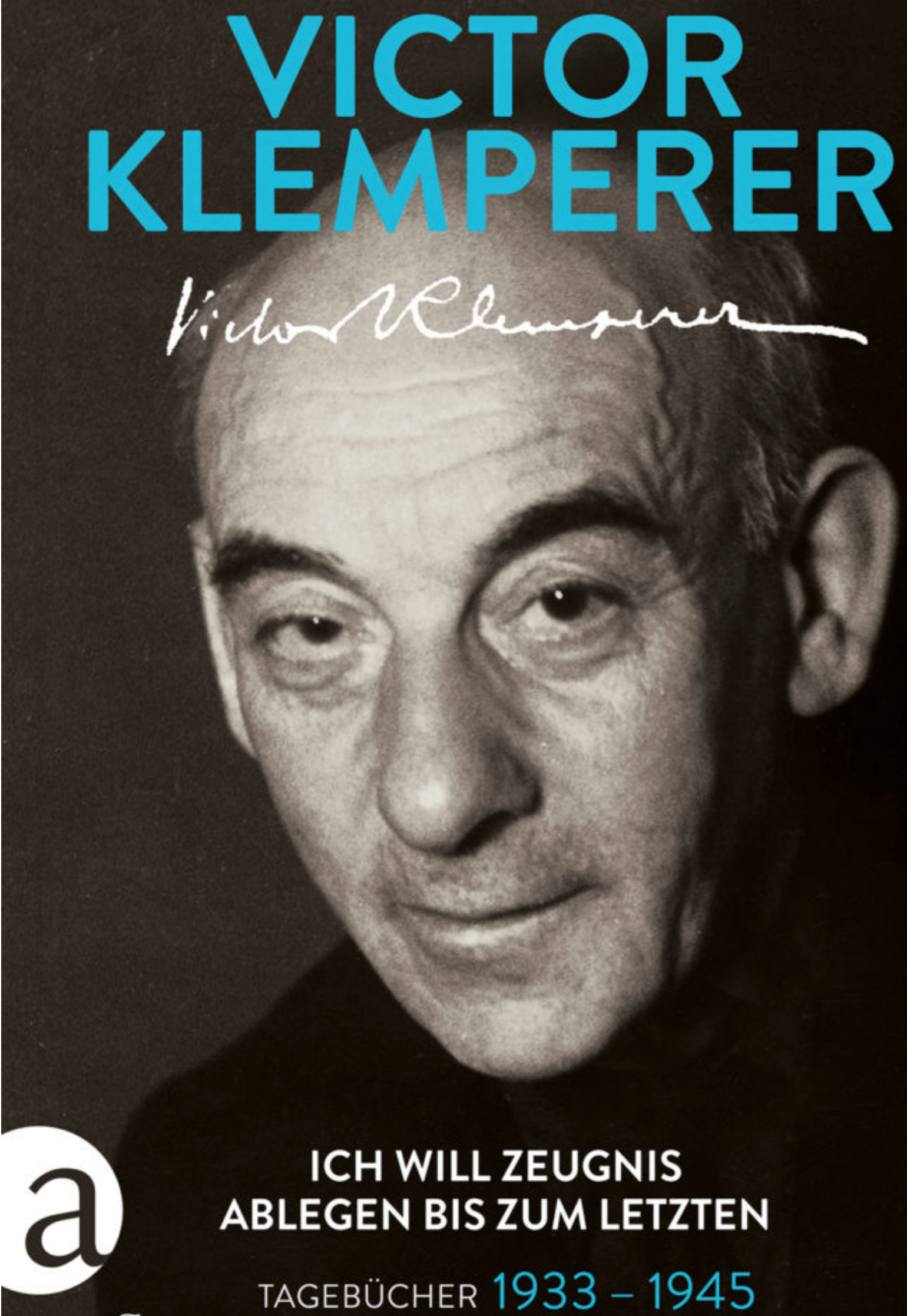

\section{ICH WILL ZEUGNIS ABLEGEN BIS ZUM LETZTEN \\ TAGEBÜCHER 1933 - 1945} aufbau 
Immagine della copertina tedesca di V. Klemperer, Testimoniare fino all'ultimo. Diari 1933-1945 edita da Aufbau, Berlino. Foto tratta da

\section{0 marzo 1933, giovedì}

Ieri a cena dai Blumenfeld[2] con i Dember.[3] L'atmosfera, quella che precedeva i pogrom nel più profondo Medioevo o nel cuore della Russia zarista. Nel corso della giornata era uscito l'appello al boicottaggio dei nazionalsocialisti. Siamo degli ostaggi. Prevale la sensazione che questo regime del terrore non durerà a lungo, ma che cadendo ci seppellirà.

\section{0 aprile 1933, giovedì sera}

È forse la suggestione di una propaganda colossale: filmati, radio, giornali, bandiere, feste sempre nuove (oggi la festa del popolo, il compleanno di Adolf, il Führer)? O è la paura da schiavi tutt'intorno che ci fa tremare?

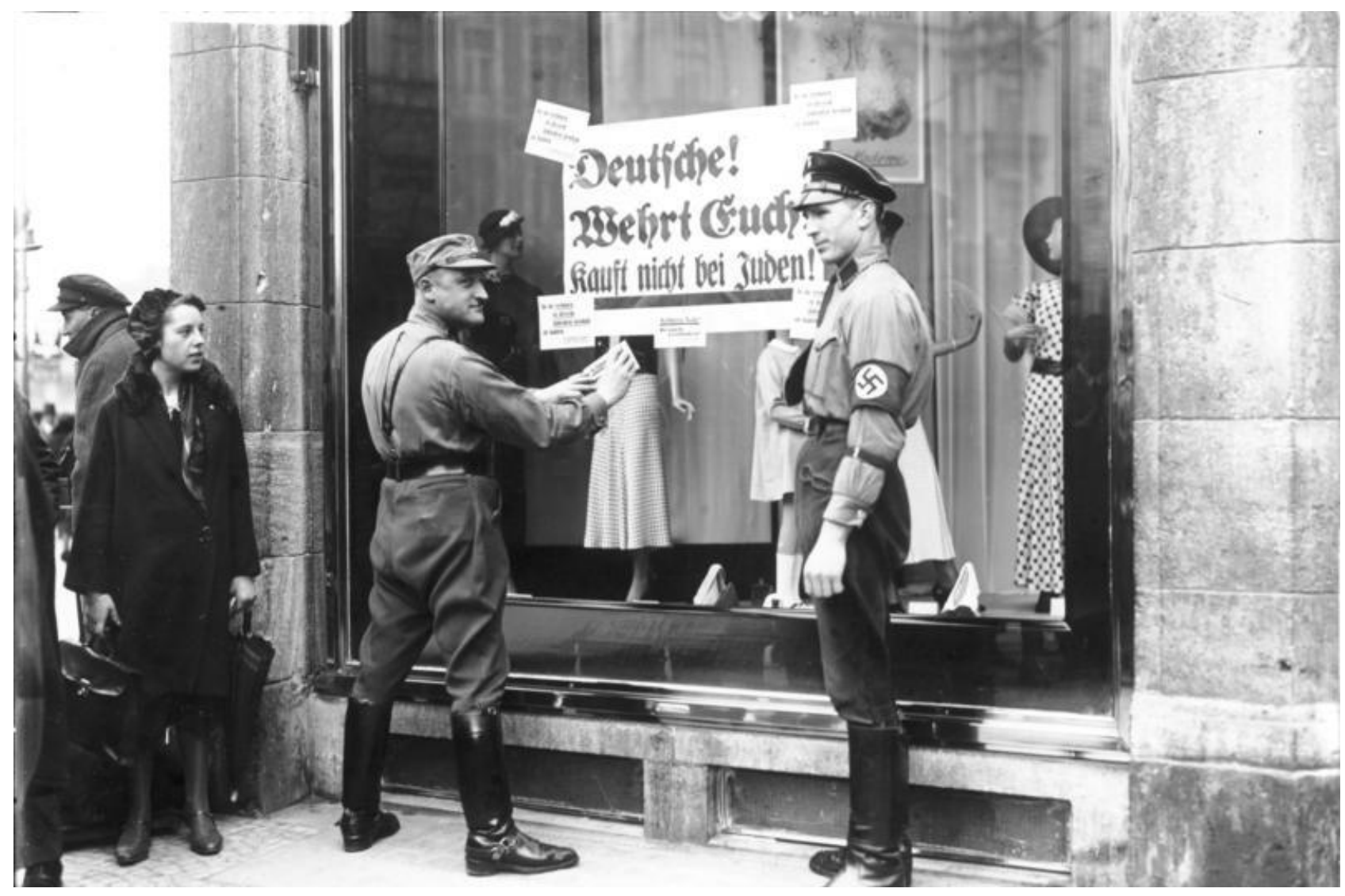

$1^{\circ}$ aprile 1933: uomini delle SA affiggono manifesti che invitano a boicottare le attività degli ebrei; sul cartello si legge: «Tedeschi difendetevi. Non comprate dagli ebrei» - Foto conservate in Bundesarchiv, Bild 102-14468 / Georg Pahl / CC-BY-SA 3.0, CC BY-SA 3.0 de, Link 


\section{4 febbraio 1934}

La signora Schaps ci ha letto delle lettere da Haifa. Sotto una coraggiosa ironia si nascondono molte difficoltà. Arduo e insensato l'apprendimento dell'ebraico - solo la bambina a scuola impara rapidamente (costretta com'è ad assimilare un'identità fittizia).

\section{$[\ldots]$}

Anche se veniamo ricacciati nel ghetto, anche se siamo calpestati e oltraggiati, e nonostante i nostri figli non possiedano più una patria se solo possiamo concludere affari, «il punto critico è stato superato». È un'idea così infinitamente indecente e spudorata che si arrivi quasi a simpatizzare con i nazionalsocialisti.

\section{2 luglio 1938, martedì, compleanno di Eva}

Mi risulta molto difficile mostrare la necessaria gioia per la ricorrenza. Questa giornata mi ricorda con troppa evidenza la miseria della nostra situazione. Lissy Meyerhof[4] scrive che Berthold ha trovato lavoro negli Stati Uniti.

Noi siamo rimasti qui nel disonore e nella miseria, in qualche modo dei sepolti vivi, sotterrati, per così dire, fino al collo, stiamo aspettando giorno per giorno le ultime badilate.

\section{9 dicembre 1939, sabato}

Lunedì sono stato alla Casa della comunità ebraica,[5] accanto alla sinagoga distrutta dalle fiamme e demolita, per pagare le tasse e il soccorso invernale. Poi il funzionario del partito che era presente mi ha voluto parlare: «L'avremmo comunque informata uno di questi giorni, entro il $1^{\circ}$ di aprile Lei dovrà lasciare la Sua casa: la può vendere, affittare o semplicemente lasciare vuota, se la veda Lei, purché se ne vada. Le spetta una stanza. Visto che Sua moglie è ariana, cercheremo di assegnargliene due». 


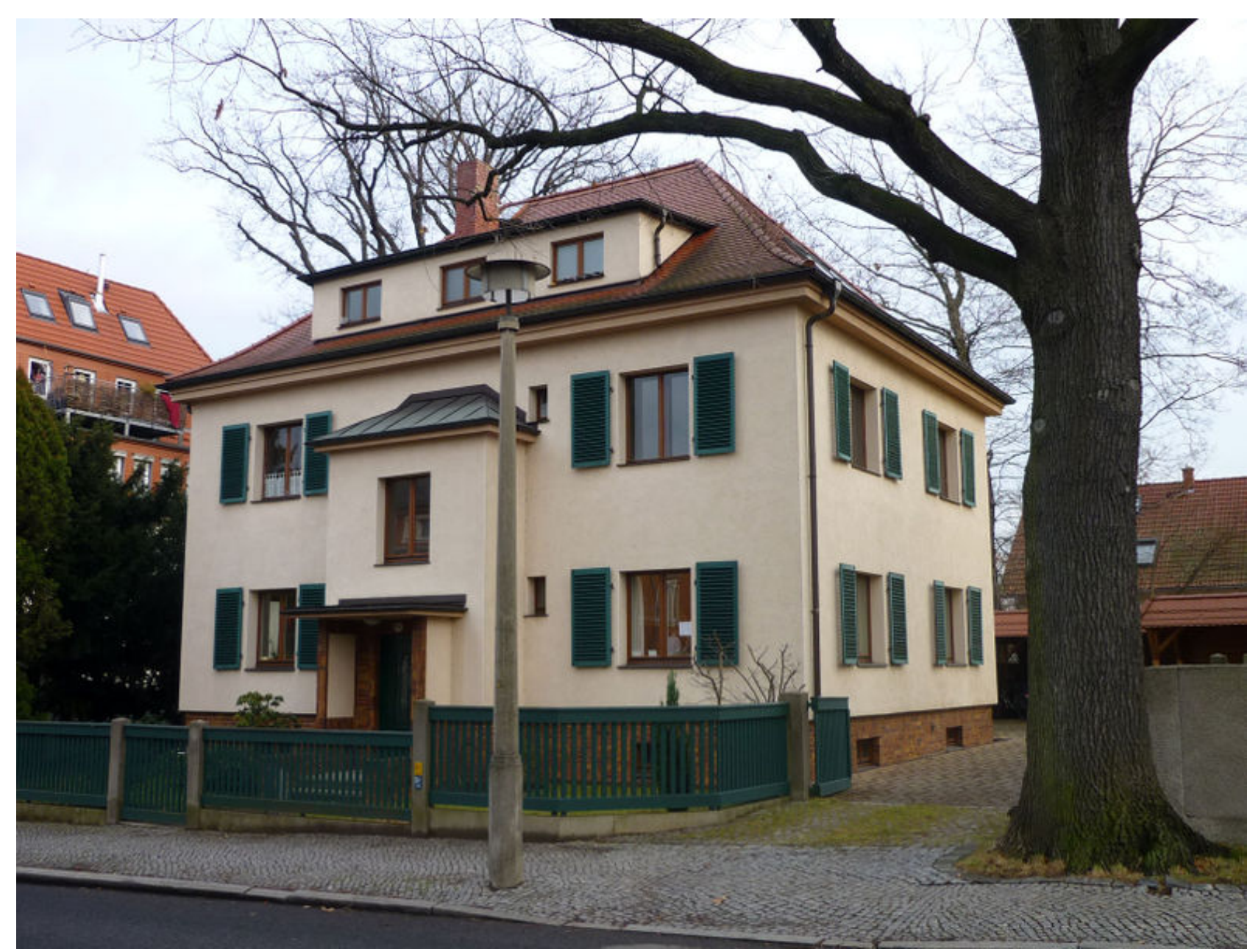

La cosiddetta «Judenhaus» sita in Caspar-David-Friedrich-Straße a Dresda, in cui abitarono i coniugi Klemperer. Foto tratta da

\section{9 aprile 1940}

Disgusto sulla questione della casa. Estreicher, che finora è stato molto gentile, ci ha mostrato sabato due stanze in una villa della Caspar-David-Friedrich-Straße.[6]

Alla mia prima domanda Estreicher[7] si è fatto estremamente arrogante, ha detto che ero irriconoscente e che avrei dovuto buttargli le braccia al collo e che dovevo decidermi subito e così via. Dopo che ci siamo scatenati a lungo l'uno contro l'altro ho accettato le due stanze. Sono stato ripetutamente messo in guardia contro Estreicher, da Neumann, da Feder, i quali dicevano che si lascia corrompere, che è una spia dei nazisti.

Non avevo intenzione di portare avanti la lite. Ho detto che gli ero assolutamente obbligato e che soltanto non riuscivo a sopportare il suo tono. Quell'uomo è un essere ripugnante, più di qualunque nazi. Eva dice che le ha fatto subito una brutta impressione, con quella linea contratta intorno alla fronte e agli occhi. 


\section{6 maggio 1940, domenica mattina}

Una villa graziosa, ma angusta e costruita in modo troppo «moderno», piena zeppa di gente che condivide tutta uno stesso destino. La villa è sistemata in modo splendido nel giardino. Un antico parco parcellizzato. Tutto questo in un bosco di lillà e castagni in fiore, la primavera si affaccia ovunque. E fin qui tutto bene; per il resto la situazione è delle più spaventose, vi sono diversi momenti nella giornata in cui vorremmo essere sotto terra. $\mathrm{E}$ frattanto oggi potrebbe cadere Calais e le prospettive di vittoria del terzo Reich sono quantomeno molto alte.

\section{8 settembre 1941}

La «stella ebraica», nera sulla stoffa gialla, sopra, in caratteri ebraicizzanti, la scritta «ebreo», va portata sul petto a sinistra, dev'essere grande quanto il palmo di una mano: ci è stata consegnata ieri per dieci pfennig, va portata da domani, 19 settembre.

\section{$[\ldots]$}

Ora la ghettizzazione è completa; in un primo tempo la parola ghetto compariva solo, per esempio, sul timbro postale «ghetto di Litzmannstadt», era riservata ai territori nemici conquistati. In Germania c'erano singole «case degli ebrei» in cui detti ebrei venivano concentrati e che talvolta venivano contrassegnate dalla scritta Judenhaus [Casa degli ebrei], ma queste case sorgevano in un quartiere «ariano» e addirittura non tutti gli inquilini erano ebrei; per cui su altre case a volte si poteva leggere l'espressione: «Questa casa è disebreizzata». Ora, dopo l'introduzione della stella gialla, non aveva più importanza se le case degli ebrei fossero sparse in giro per la città o riunite in un solo quartiere, perché ogni ebreo, con la stella, portava con sé il proprio ghetto, come la chiocciola la sua casa.

\section{4 marzo 1942, martedì}

Quello che mi pesa di più è la mattinata. Soffro il freddo (nel locale non riscaldato), sono affamato e per la stanchezza mi addormento spesso alla scrivania. Allora cerco di rubare un cucchiaio di marmellata o un pezzettino di pane in cucina, da Kätchen Sara,[8] ma lo posso fare solo se ce n'è abbastanza perché lei non debba notare nulla. E comunque sono sempre preoccupato che mi possa sorprendere.

Anche di fronte a Eva conservo il mio penoso segreto.

\section{3 maggio 1942, sabato pomeriggio}

Ieri mattina la notizia della morte di Ernst Kreidl, nel pomeriggio la tanto attesa perquisizione. Alle quattro e un quarto sono andato ancora una volta (molto malvolentieri) da Steinitz - i soliti discorsi. Alle sette e mezzo sono rientrato. La squadraccia era comparsa alle cinque e se ne era andata poco prima del mio rientro. Prima di tutto ho visto, dalla porta d'entrata aperta, il caos al pian terreno. La signora Kreidl e la signora Pick erano state picchiate. Da noi ho trovato Eva perfettamente padrona di sé. Diceva che tutto s'era svolto come previsto. «Sei ariana? - Ehi, puttana degli ebrei, perché hai 
sposato un ebreo? Nel Talmud sta scritto: “Ogni donna non ebrea per noi è una puttana”...» L'hanno mandata di sotto. Giù si è presa un paio di ceffoni - «Più teatrali che altro».

\section{7 maggio 1942, mercoledì a mezzogiorno}

Oggi pomeriggio Eva va a Pirna a prendere un po' di soldi. Le do da portare le pagine di diario delle ultime settimane. Dopo la perquisizione ho trovato sul tavolo alcuni libri che erano stati presi dallo scaffale: se il vocabolario di greco fosse stato tra loro, se ne fossero caduti fuori i fogli di diario e avessero suscitato un sospetto, sarei andato incontro a morte certa. Si viene uccisi per molto meno...Così questi frammenti oggi se ne vanno.

\section{7 maggio 1942, mercoledì a mezzogiorno}

Eppure io continuo a scrivere. È questo il mio modo di essere eroico.

\section{2 giugno 1942, martedì verso sera}

Quante se ne sono viste in questi ultimi anni, di grandi e piccole! E talvolta una puntura di spillo è anche più dolorosa del colpo di clava. Ora, per una volta, riassumo le disposizioni: 1) Dopo le otto o le nove di sera bisogna essere a casa. 2) Buttati fuori di casa. 3) Divieto di possedere una radio, divieto di possedere il telefono. 4) Divieto di andare a teatro, al cinema, al concerto, al museo. 5) Divieto di abbonarsi o acquistare riviste. 6) Divieto di viaggiare: a) vietati gli autobus, concessa soltanto la piattaforma anteriore del tram; b) vietato in genere viaggiare con i mezzi pubblici, a meno che non li si utilizzi per andare al lavoro; c) anche al lavoro a piedi, a meno che non si abiti a più di $7 \mathrm{~km}$ di distanza o si sia malati. Naturalmente è vietato anche il taxi. 7) Divieto di acquistare «merce rara». 8) Divieto di acquistare sigari e ogni genere di tabacchi. 9) Divieto di acquistare fiori. 10) Sottratta la tessera per il latte. 11) Divieto di recarsi dal barbiere. 12) Divieto di acquisto presso gli artigiani: per ogni cosa è necessario presentare una richiesta alla Comunità. 13) Obbligo di consegnare le macchine da scrivere. 14) Consegna di pellicce e coperte di lana. 15) Consegna di biciclette 16) Consegna delle sedie a sdraio. 17) Consegna di cani, gatti, uccelli. 18) Divieto di lasciare il territorio urbano di Dresda. 19) Divieto di entrare in stazione. 20) Divieto di recarsi sulla riva del Ministero e nei parchi. 21) Divieto di entrare nei mercati. 22) Dal 19 settembre la stella ebraica. 23) Divieto di tenere in casa provviste di cibo 24) Divieto di frequentare le biblioteche circolanti. 25) Con la stella ci sono preclusi tutti i ristoranti. 26) Niente tessere per il vestiario. 27) Niente tessere per il pesce. 28) Niente assegnazioni speciali come caffè, cioccolata, frutta o latte condensato. 29) Le tasse supplementari. 30) La franchigia che si restringe sempre più. La mia dapprima 600, poi 320, ora 185 marchi. 31) Restrizione dell'orario per gli acquisti a una sola ora al giorno (dalle tre alle quattro, il sabato dalle dodici all'una).

Credo che in questi 31 punti vi sia tutto. Ma sommati non sono nulla rispetto al costante pericolo delle perquisizioni, dei maltrattamenti, della prigionia, dei campi di concentramento e della morte violenta.

Ora viviamo letteralmente di elemosine. [...] 


\section{7 luglio 1942, Sera}

Kätchen racconta: l'istituto Henriette, circa cinquanta anziani, viene evacuato. Ed ecco che sua madre, quell'indistruttibile ottantenne, deve partire anche lei per Theresienstadt; il fratello di Kätchen è stato arrestato e quindi è un uomo morto. Questo Joachimsthal è un pessimo soggetto, con il quale lei ha già litigato e che la ricatta - ma perché ora viene ucciso?

\section{6 agosto 1942, giovedì mattina}

Abitudine: sono passate un paio di settimane dall'assassinio di Joachimsthal,[9] un paio di mesi dalle perquisizioni. E io già vivo in uno stato di quiete vicino all'apatia. Abitudine: martedì parte un altro trasporto da qui per Theresienstadt; e già mi sembra, sembra a tutti gli ebrei qui, una cosa ovvia.

\section{4 agosto 1942}

Ieri con Eva dai Marckwald. Abbiamo incontrato là un uomo secco, sui cinquant'anni, commerciante di granaglie, ora infermiere della Comunità ebraica: Bernstein. Quell'uomo, di fronte al paralitico Marckwald, si è espresso in toni ancora più disperati di quelli usati recentemente da Katz sull'ultimo trasporto. I paralitici più gravi stipati come sardine sulle panche all'interno del camion, una guida tutta a scossoni e senza riguardi, persino mentre venivano praticate le iniezioni, mancavano i medicinali, per andare a prelevare la gente dalle case non erano consentite le ambulanze, portarsi le sedie a rotelle escluso e così via...

Ce ne siamo andati insieme e io gli ho chiesto perché avesse fatto una descrizione così raccapricciante. Risposta: detto a quattr'occhi, lui a Marckwald consiglierebbe senz'altro di suicidarsi. La brutalità del trasporto è inconcepibile. Non c'è riguardo per nessuno, né per l'età, né per la paralisi, anche se completa, né per il dolore. Ciò che in tutto questo mi sembra superare di gran lunga analoghi orrori perpetrati dai russi è: non vi è nulla di spontaneo qui, tutto è organizzato e sistemato con metodo, è un orrore «colto», e si finge che tutto accada in nome della cultura e non si fa che mentire. Da noi non si uccide.

\section{4 dicembre 1943}

La promiscuità. È davvero una vita da baraccati, si inciampa gli uni sugli altri, confusione. E tutti gli ebrei ammucchiati. Quando sono andato in cantina dall'amministratore Waldmann a prendere una scodella, ho incontrato il barbiere degli Schuster, Friedheim e sua moglie, Steinitz junior, che gioca al capomastro da Bauer e che so io.[10] - L'aspetto pubblico e promiscuo di questa vita mi sembra la cosa peggiore. 
[...] Conversazione con Stühler senior: «Voglio testimoniare.» - «Quello che scrive Lei sono tutte cose note, mentre i grandi eventi, Kiev, Minsk ecc., Lei non li conosce.» - «Il problema non sono i grandi eventi, ma la tirannia nella sua quotidianità, che verrà dimenticata. Migliaia di punture di zanzara sono peggio di un colpo sulla testa. Io osservo e prendo nota delle punture di zanzara...»

\section{4 settembre 1944, giovedì mattina}

$[\ldots]$

Siamo travolti dal presente, non esiste più una suddivisione temporale, tutto è accaduto un'infinità di tempo fa, ogni cosa si fa attendere all'infinito, non c'è ieri né domani, solo un'eternità.

\section{7 settembre 1944, mercoledì mattina}

Oggi pomeriggio Eva intende andare a Pirna.

I miei diari e le mie annotazioni! Mi continuo a dire: non ne va soltanto della mia vita se qualcuno li scopre, ma della vita di Eva e di parecchi altri che ho citato per nome, che ho dovuto citare per nome se voglio che i diari acquistino un valore documentario. Ne ho il diritto, sono tenuto a farlo, o si tratta di una forma criminale di presunzione?

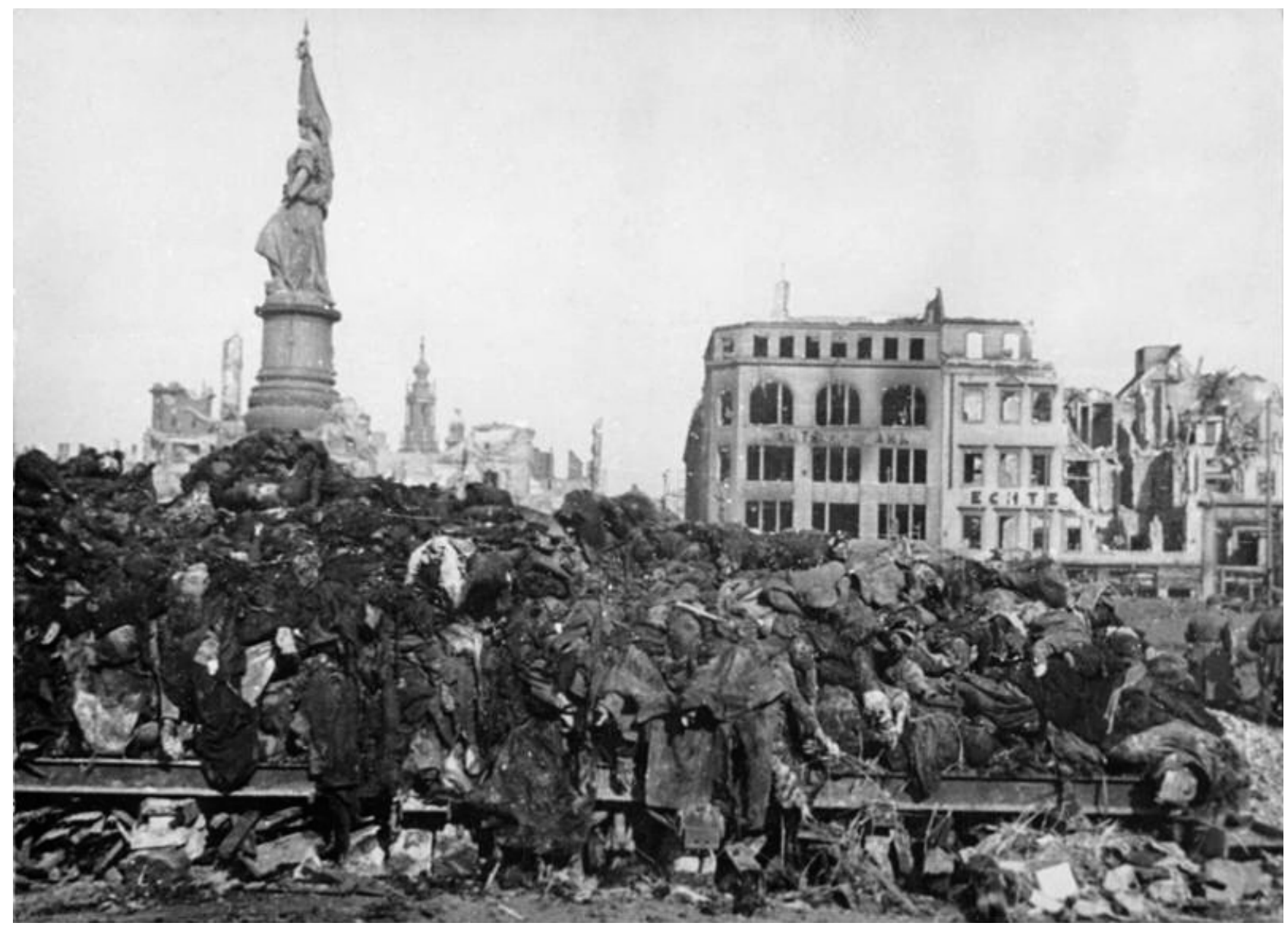


Montagna di cadaveri al Mercato vecchio di Dresda, febbraio 1945 - Von Bundesarchiv, Bild 18308778-0001 / Hahn / CC-BY-SA 3.0, CC BY-SA 3.0 de, https://commons.wikimedia.org/w/index.php?curid=5420481

\section{Distruzione di Dresda, 13-14 febbraio 1945}

A volte sul cammino giacevano, sparsi qua e là, dei cadaveri: piccoli fagotti, nient'altro che un mucchio di abiti. Uno aveva il cranio fracassato e la parte superiore della testa era una scodella rosso cupo.

C'era un braccio con una mano pallida, non brutta, come quegli arti modellati con la cera che si vedono nelle vetrine dei parrucchieri.

\section{6-17 febbraio 1945}

A Klotzsche ho cominciato per la prima volta a riflettere su quanto era andato perduto [nella distruzione di Dresda]. Tutti i miei libri, i dizionari, le mie stesse opere. Se a Pirna capita una disgrazia, tutto il mio lavoro, a partire dal 1933, va distrutto. Ogni volta che pensavo e penso a quei mucchi di macerie, avevo e ho anch'io quel sentimento atavico: Jahve!

\section{Monaco, 22 maggio 1945}

Qui tutto è distrutto, mostruosi cumuli di macerie bloccano il cammino, rovine cadenti, travi sospese che fluttuano irreali. [...] Alla Feldherrnhalle, c'è una scritta dipinta con cura a lettere cubitali: «Buchenwald, Velden, Dachau - mi vergogno di essere un tedesco». Lì vicino, su un portone, più in piccolo, ci sono queste parole: Au pays des Crématoires.

\section{Dresda, sabato mattina, 21 luglio 1945}

Tutto è fermo, tutto vacilla. Il tempo scorre tra la calura, i disturbi di cuore, il pane secco. Gradevole, in tutto questo, solo il torpore che dà l'ascolto protratto della radio. - 


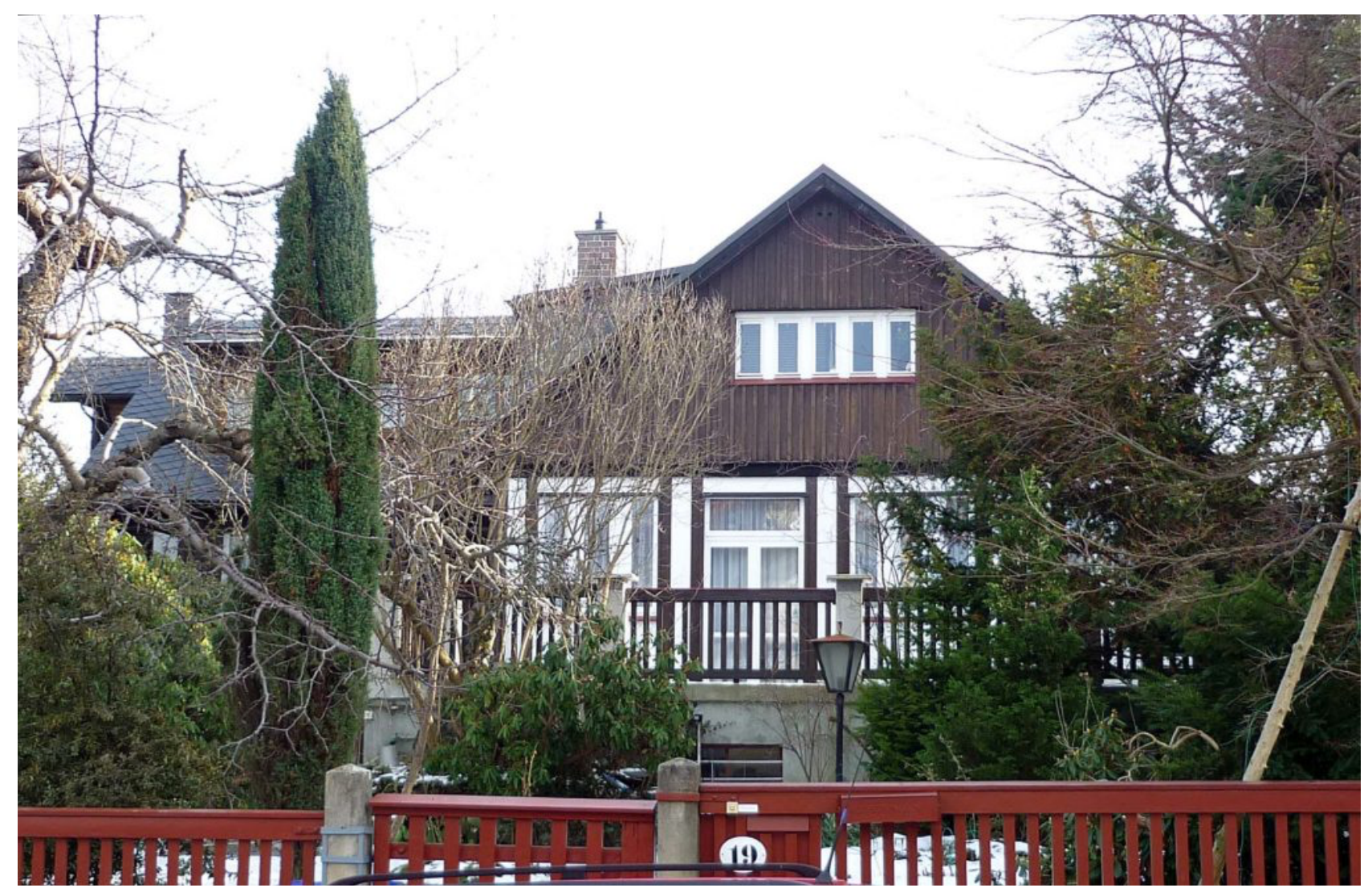

La casa dei Klemperer a Dresda-Dölzschen.

Von SchiDD - Eigenes Werk, CC BY-SA 3.0, Link

\section{martedì 19 giugno 1945, Dölzschen}

Siamo arrivati qui domenica 10. La prima notte abbiamo dormito dal buon Kalau, la seconda già in casa nostra, una sensazione che continua a essere indescrivibile, come un sogno a occhi aperti, viviamo da allora in un mondo di favola, un mondo ridicolo, immaginario eppure terribilmente reale, un paradiso, a volte commovente, a volte un po' spregevole, e ancora non ho avuto la calma necessaria per poter lavorare, i miei diari languono, non ho ancora completato i pochi appunti delle giornate di viaggio, non ho fissato su carta tutto quello che è accaduto in questa

settimana, non mi sono ancora occupato del destino dei miei manoscritti a Pirna,[11] non mi sono ancora esercitato seriamente sulla macchina da scrivere, ogni uscita una grande occasione che riempie la giornata (circostanza attenuante in questo senso il mio piede gonfio o e il problema del traffico), la mia unica lettura finora è il volumetto Giochi africani di Ernst Jünger (per molte cose di una qualità sorprendente, per altre incomprensibile): sono troppo stanco per qualunque cosa. Le giornate trascorrono mangiando, e di nuovo mangiando, sonnecchiando e dormendo, e poi ci sono le visite, ne ricevo e faccio molte, le chiacchiere, i progetti e lo scetticismo, lo stupore, la gioia e di nuovo lo scetticismo, la stanchezza, l'attesa, il lasciare che le cose vadano come devono andare, e di nuovo la stanchezza. Oscillo di continuo tra una beatitudine piena di stupore e la sorpresa incredula per questa svolta fiabesca del nostro destino e la paura oscura che tutto questo sia arrivato troppo tardi, il cuore, la senescenza del pensiero, anche il semplice arrugginirsi delle mie conoscenze - non so più nemmeno dieci parole di francese - potrebbero giocarmi un brutto tiro e distruggermi. Ma tutto questo, speranza, paura, scetticismo, angoscia, è attutito dalla stanchezza e da una pigra 
sensazione animalesca di benessere: questo continuo mangiare, dormire, e tra l'una e l'altra cosa ascoltare un po' di radio.

Comunque: uno dei primi giorni sono andato da Neumark, poi da Katz e ieri - decisivo! O forse no?

Oh Repubblica dei Consigli! Oh ricordo del ministro Landauer! - da Grohmann, alla

Melanchthonstr. Avevamo immaginato spesso come sarebbe stato nel momento in cui fossimo davvero tornati a casa. Provavo un senso di disgusto all'idea di dover avere ancora a che fare con queste persone. Eva, che ha un altro tipo di rapporto con questa casa (e la cui felicità dalla mattina alla sera, il cui rifiorire, addirittura da un'ora all'altra, sono per me la gioia più grande in tutta questa faccenda), Eva, ha detto che non dovremmo occuparci di nessuno e che non dovremmo far altro che starcene nel bozzolo del nostro giardino. Ma non è cosi: siamo costantemente, e fin dal primo istante, al centro di un'accoglienza trionfale e non facciamo che oscillare tra la commozione e il disprezzo per il genere umano. Alcune persone sono sicuramente sincere, ma le altre??? La storia è cominciata proprio quando siamo arrivati quassù, otto giorni fa, di pomeriggio. Una giovane donna ci ha rincorso: abbiamo parlato così spesso di voi, ancora ieri ne abbiamo parlato! Né io né Eva la conoscevamo, la moglie del dottor König un medico dentista. Abbiamo dovuto bere il caffè da lei, ci ha sommerso di sigarette e di altre attenzioni. Nel frattempo ci è venuta a trovare, ha portato marmellata e altro cibo, abbiamo conosciuto anche suo marito. L'impressione non è male però, però. Il sindaco non siamo riusciti a incontrarlo, la stessa signora König ci aveva indicato Kalau, marito della levatrice in Pesterwitzerstr., come persona particolarmente potente.

L'ho chiamato, gli ho detto che venivo da parte del dott. König, mi ha risposto bruscamente che non era in servizio. Ma non appena ha sentito il mio nome e di cosa si trattava, si è fatto molto cortese e di una cordialità che finora si è dimostrata sincera e costante. L'errore poi si è chiarito: Kalau è colui che assegna gli incarichi del servizio del lavoro,[12] cioè che fa spalare i Pg.[13] E per questo è costantemente sommerso di reclami e di richieste. Tutti ammettono solo se costretti di essere stati Pg. Tra coloro che quel giorno erano stati impegnati per la prima volta nel servizio c'era il dott. König, cosa che io ovviamente non potevo sapere, e che naturalmente mi fa apparire un po' sospetta l'estrema gentilezza dimostrataci con tanta solerzia dalla famiglia. Tuttavia quell'uomo mi ha dato l'impressione di una persona totalmente estranea alla politica che si è davvero iscritta al partito soltanto per poter continuare a esercitare il proprio lavoro, un po' come Johannes Köhler. (Ma quei milioni di dott. König e di prof. Köhler - non sono forse proprio loro i veri colpevoli ????)

\section{Nota bibliografica}

Le opere di Klemperer finora tradotte in italiano sono: LTI. La lingua del Terzo Reich. Taccuino di un filologo, nuova edizione riveduta e annotata a cura di Elke Fröhlich, trad. di Paola Buscaglione Candela, prefazione di Michele Ranchetti, Giuntina 2011; Testimoniare fino all'ultimo. Diari 19331945, edizione italiana a cura di Anna Ruchat e Paola Quadrelli, prefazione di Cesare Segre, Mondadori 2000; E cosi tutto vacilla. Diario del 1945, a cura di Anna Ruchat, prefazione di Antonio Moresco, Scheiwiller 2010.

Un importante strumento di approfondimento è il sito Klemperer on line https://www.degruyter.com/view/db/klemp

(in tedesco e in inglese) che raccoglie i diari 1918-1959 e molti altri elementi di ricerca, comprese le riproduzioni degli originali dei diari e un ampio apparato bibliografico. 
Le opere di Klemperer sono state tradotte in molte lingue e in tedesco sono disponibili anche diverse edizione audio dei suoi diari. Per la televisione tedesca è stata prodotta nel 1999 una serie in 12 episodi dal titolo Klemperer - Ein Leben in Deutschland, (regia di Kai e Andreas Kleinert) che ripercorre la storia di Klemperer nel regime nazista, a partire dai suoi diari. Dal 2010 la serie è disponibile in Dvd.

\section{Note:}

[1] A. Ruchat (a cura di), E cosi tutto vacilla. Diario del 1945 (prefazione di Antonio Moresco), Milano, Scheiwiller 2010, p. 8.

[2] Walter Blumenfeld, (1882-1967), psicologo; dal 1924 al 1934 professore di pedagogia presso la TH di Dresda; nel 1935 emigra in Perù dove ottiene una cattedra di psicologia sperimentale e pedagogica presso l'università di Lima. Con la moglie Grete era tra gli amici più intimi dei Klemperer.

[3] Harry Dember, (1882-1943), fisico, dal 1923 professore alla TH di Dresda. Nel1933 emigrò con la famiglia a Istanbul, nel 1941 negli Usa. Lui e sua moglie Agnes, che tornò più volte a Dresda tra il 1933 e il 1937 perché la madre era rimasta in Germania, erano molto amici dei Klemperer.

[4] Lissy Meyerhof, infermiera, educatrice, amica dei Klemperer, viveva a Berlino, è deportata il 25 gennaio 1942 e dichiarata dispersa.

[5] La comunità ebraica, a Dresda come in altre città tedesche, faceva da intermediaria, con non poche ambiguità, tra il regime e i singoli cittadini ebrei.

[6] Dal maggio del 1940 al settembre del 1942, i Klemperer hanno abitato nella Casa degli ebrei di Caspar-David-Friedrich-straße, che fu il loro secondo domicilio coatto.

[7] Funzionario della comunità ebraica di Dresda.

[8] Kätchen Sara Voß (1882-1943), nata Joachimsthal vedova di un assicuratore, coinquilina dei Klamperer. In Caspar-David-Friedrich-Straße, e nella Altenzellerstraße, nel 1943 lavorava per la Zeiß-Ikon di Dresda. Fu deportata ad Auschwitz con l'ultimo grande trasporto degli ebrei di Dresda dal campo di Hellerberg e uccisa subito dopo il suo arrivo.

[9] Joachimsthal, giornalista, cugino di Kätchen Sara Voß. Così lo ritraeva Klemperer. nei Diari del 1943 (29 aprile): «I compagni di lavoro del turno di notte: alla macchina Joachimsthal, sui cinquantacinque, cugino di Kätchen Sara, acculturato ma non troppo, proviene dal commercio librario e dal giornalismo, uno psicopatico grave, diffidente, sovreccitato, continua a sospirare e a prendersela per ogni cosa, si sente sempre aggredito e trascurato. Di me - per ora - si fi da, con gli altri litiga, arriva persino, spudorato com'è, a invocare il caposquadra ariano». Muore per i pestaggi in carcere nel 1943.

[10] In italiano nel testo. 
[11] Annemarie Köhler, amica die Kl. Fin dal loro soggiorno a Lipsia nel 1918, ariana, chirurgo presso l'ospedale del Sacro Cuore a Pirna, conservò i manoscritti e i diari di Kl. a partire dal 1939 e fino alla fine della guerra.

[12] L'Arbeitsdienst [servizio del lavoro] era un'istituzione del Terzo Reich che impiegava le persone (in particolare gli ebrei, i disoccupati o altre classi di emarginati) con scopi di utilità "pubblica". Klemperer aveva spalato la neve a Dresda nell'inverno del 1943 e del 1944. Cfr. Aldo Enzi, Il lessico della violenza nella Germania nazista: L'Arbeitsdienst «era definito "la grande scuola educativa della comunità nazionalsocialista» e pertanto era un servizio d'onore».

Nell'immediato dopoguerra il «servizio del lavoro» a Dresda consiste nella rimozione delle macerie e conserva un carattere punitivo, questa volta nei confronti degli ex Pg.

[13] Pg sta per Parteigenosse, ex appartenente al partito nazionalsocialista. 